\title{
25 Research Sourere \\ CRISPR/Cas9-mediated Targeted Mutagenesis in Japanese Cedar (Cryptomeria japonica D. Don)
}

\section{Yoshihiko Nanasato ( $\square$ nanasato@affrc.go.jp )}

Forest Bio-Research Center, Forestry and Forest Products Research Institute

\section{Masafumi Mikami}

Graduate School of Nanobioscience, Yokohama City University

\section{Norihiro Futamura}

Forestry and Forest Products Research Institute

\section{Masaki Endo}

Institute of Agrobiological Sciences, National Agriculture and Food Research Organization

\section{Mitsuru Nishiguchi}

Forestry and Forest Products Research Institute

\section{Yasunori Ohmiya}

Forestry and Forest Products Research Institute

\section{Ken-ichi Konagaya}

Forest Bio-Research Center, Forestry and Forest Products Research Institute

\section{Toru Taniguchi}

Forest Bio-Research Center, Forestry and Forest Products Research Institute

\section{Research Article}

Keywords: Cryptomeria japonica, tree species , breeding programs, Genome editing technology

Posted Date: March 11th, 2021

DOI: https://doi.org/10.21203/rs.3.rs-275612/v1

License: (c) (1) This work is licensed under a Creative Commons Attribution 4.0 International License. Read Full License

Version of Record: A version of this preprint was published at Scientific Reports on August 10th, 2021. See the published version at https://doi.org/10.1038/s41598-021-95547-w. 


\section{Abstract}

Cryptomeria japonica (Japanese cedar or sugi) is one of the most important coniferous tree species in Japan and breeding programs for this species have been launched since 1950s. Genome editing technology can be used to shorten the breeding period. In this study, we performed targeted mutagenesis using the CRISPR/Cas9 system in C. japonica. First, the CRISPR/Cas9 system was tested using green fluorescent protein (GFP)-expressing transgenic embryogenic tissue lines. Knock-out efficiency of GFP ranged from 3.1-41.4\% depending on U6 promoters and target sequences. The GFP knock-out region was mottled in many lines, indicating genome editing in individual cells. However, in 102 of 103 mutated individuals (>99\%) from 6 GFP knock-out lines, embryos had a single mutation pattern. Next, we knocked out endogenous $C$. japonica magnesium chelatase subunit I (CjCh/I) using two guide RNA targets. Green, pale green, and albino phenotypes were obtained in genome-edited cell lines. Sequence analysis revealed random deletions, insertions, and replacements in the target region. Thus, targeted mutagenesis using the CRISPR/Cas9 system can be used to modify the $C$. japonica genome.

\section{Introduction}

Cryptomeria japonica D. Don (Japanese cedar or sugi) is an evergreen conifer, which belongs to the cypress family (Cupressaceae) and is distributed across Japan. Outside Japan, C. japonica var. sinensis (formerly $C$. fortune $I$ ) is thought to be naturally distributed in southeast China ${ }^{1}$. C. japonica is a commercially important tree in Japan and has been used since ancient times as timber and to produce household items, etc. because of its high productivity and utility. C. japonica cultivation began more than 500 years ago in some areas of $\mathrm{Japan}^{2}$. A breeding program has been in progress since 1950s for screening plus trees; thus far, more than 3,500 plus $C$. japonica tree clones have been selected ${ }^{3}$. $C$. japonica was extensively planted after WWII because of the government's efforts to restore devastated forests. Consequently, C. japonica comprises $44 \%$ of the planted forest area in Japan, which covers approximately $11 \%$ of the land in $\mathrm{Japan}^{4}$. C. japonica pollinosis has become a severe public health problem in Japan due to the widespread distribution of $C$. japonica pollen ${ }^{5}$. Development of "male-sterile" C. japonica trees is a breeding target in Japan. Studies for identifying genes involved in pollen development are underway ${ }^{6,7}$. Four male-sterility loci, namely $M S 1, M S 2, M S 3$, and $M S 4$, have been identified from 23 male-sterile $C$. japonica lines $^{8}$, and Hasegawa et al. elucidated a causative male-sterile gene at the MS1 locus ${ }^{9}$. Additionally, SNP markers for distinguishing MS1-derived male-sterile plants have been developed ${ }^{10}$. Recently, an efficient technique for producing male-sterile $C$. japonica plants using somatic embryogenesis was reported ${ }^{11}$.

To obtain the desired trait through gene modification in a short time, programmable nuclease-mediated targeted mutagenesis is the option of choice and several methods, such as zinc-finger nuclease (ZNF), transcription activator-like effector nuclease (TALEN), and clustered regularly interspaced short palindromic repeat (CRISPR)/CRISPR-associated protein 9 system (CRISPR/Cas9), have been developed. The use of CRISPR/Cas9 system, first reported in $2012^{12}$, has exploded because of its efficiency and 
simplicity, and has been applied to at least 45 plant genera ${ }^{13}$. The CRSIPR/Cas 9 system has been improved by expanding the targeting range of Cas $9^{14,15}$ and developing smaller Cas proteins ${ }^{16,17}$. In addition, genome editing systems based on the CRISPR/Cas9 system without involving double-strand breaks, such as base editing ${ }^{18,19}$ and prime editing ${ }^{20}$ have been reported. Among woody plants, CRISPR/Cas9-mediated targeted mutagenesis in Populus, which is a model woody species, was first reported in $2015^{21,22}$. However, the use of genome editing in conifers remains unexplored.

In this study, we demonstrated CRISPR/Cas9-mediated targeted mutagenesis in $C$. japonica through Agrobacterium-mediated transformation. First, we targeted the exogenous green fluorescent protein (GFP) gene, which was introduced into the cell mass of embryonic tissue, after examining various promoters to construct an efficient targeted mutagenesis system in C. japonica. Targeted GFP knock-out was observed in transgenic lines at a constant rate with the CRISPR/Cas9 expression vectors. Then, as a case study, we disrupted the endogenous magnesium chelatase subunit I (CjChII) gene using a modified vector and obtained biallelic mutant lines. Thus, the CRISPR/Cas9 system is efficient in $C$. japonica. We discuss future perspectives in genome editing in conifers.

\section{Materials And Methods}

\section{Plant material}

An embryogenic tissue (ET) cell line \#13-8-12 was initiated using megagametophyte explants of $C$. japonica ${ }^{23}$. The ET was maintained in the dark at $25^{\circ} \mathrm{C}$ in solid $1 / 2 \mathrm{MD}$ medium ${ }^{24}$ and subcultured on fresh media after a 2-week interval.

\section{Vector construction}

The GFP expression vector pZmUbi-GFP-Dt (Fig. 1a) was constructed based on pUbiP-sGFP/HygR ${ }^{23}$. The NPTIl expression cassette was removed from pUbiP-sGFP/HygR using Pmel and Clal followed by blunting with the KOD Plus polymerase (Toyobo, Osaka, Japan) and self-ligation using T4 DNA ligase (New England Biolabs, Ipswich, MA). The heat shock protein terminator from Arabidopsis thaliana ${ }^{25}$ was inserted downstream of GFP using In-Fusion cloning (In-Fusion HD Cloning Kit, Takara Bio Inc., Shiga, Japan). pZK_FFCas9 (Fig. 1b) was constructed according to Mikami et $\mathrm{al}^{26}$. Briefly, the PcUbi::SpCas9::PsBCB3At fragment from pDeCas927 and the OsAct1 t::35S::NPTII::OsHSP17.3t fragment were cloned into $\mathrm{pZK}^{28}$ using In-Fusion cloning. The gRNA expression cassette driven by the $A$. thaliana U6-26 promoter, derived from pEn-Chimera ${ }^{27}$, was cloned into the multiple-cloning site of pUC19. To use the OsU6 promoter, pUC6gRNA ${ }^{29}$ was used. U6 promoters from $C$. japonica were replaced with the OsU6 promoter in pUC6gRNA (described in Supplementary Information). All oligonucleotides used in this study are shown in Supplementary Table S1. Oligonucleotide pairs for target GFP sequences ( $p \# 84$ and $p \# 85$ for target \#1, p\#86 and p\#87 for target \#2_rev, and p\#88 and p\#89 for target \#3_rev) (Table 1, 
Supplementary Table S1) were annealed, and the resulting fragment was inserted into $B b s l$ sites between the U6 promoter and gRNA scaffold sequence of the arbitrary gRNA expression cassette. The gRNA expression cassette was inserted into the I-Scel site between the right border and the ubiquitin promoter from Petroselinum crispum (PcUbi) ${ }^{30}$ in pZK_FFCas9, resulting in the all-in-one CRISPR/Cas9 vector (Fig. 1b). For targeted mutagenesis of endogenous CjChll, the modified CRISPR/Cas9 vector, namely pCRGSpCas9 (Fig. 1C), was constructed. Briefly, the HSP terminator from $A$. thaliana and NOS terminator were tandemly inserted downstream of FFCas9. The gRNA expression cassette was inserted into the Ascl site between the right border and PcUbi in the pCRG-SpCas9 vector through overlapping $\mathrm{PCR}^{31}$. The detailed method for vector construction is described in Supplementary Information.

Table 1

Target sequence of GFP

\begin{tabular}{|lll|}
\hline Target & Sequence $\left(\mathbf{5}^{\prime} \mathbf{- 3}^{\prime}\right)$ & Reference \\
\hline$\# 1$ & GTGAACCGCATCGAGCTGAAGGG & Mali et al (2013) \\
\hline$\# 2$ rev & CCTACGGCGTGCAGTGCTTCAGC & Jacobs et al (2015) \\
\hline \#3_rev & CCACCGGCAAGCTGCCCGTGCCC & Fu et al (2013) \\
\hline
\end{tabular}

\section{Transformation of $\boldsymbol{C}$. japonica}

ET cell lines expressing GFP were generated through the Agrobacterium-mediated transformation of pZmUbi-GFP-Dt/HygR ${ }^{24,32}$. After screening with $5 \mathrm{mg} / \mathrm{L}$ hygromycin, lines with a strong GFP signal with a single-copy line (named N4) were selected through Southern blot analysis (Supplementary Fig. S1). The N4 line was transformed with pZK_FFCas9 vectors containing various gRNA expression cassettes. Transformed cell lines were selected on $1 / 2 \mathrm{MD}$ medium containing $10 \mathrm{mg} / \mathrm{L}$ meropenem and $25 \mathrm{mg} / \mathrm{L}$ kanamycin. Transformed cell lines were maintained at $25^{\circ} \mathrm{C}$ under dark. Somatic embryogenesis and germination were performed as described ${ }^{24}$.

\section{GFP visualization}

After screening for 2-4 months in the selection medium, independent lines were isolated and considered a single event. GFP fluorescence of independent lines was evaluated using the MS FLIII fluorescence stereomicroscope (Leica microsystems, Wetzlar, Germany) with a GFP Plus filter system (excitation filter $480 / 40 \mathrm{~nm}$, emission filter $510 \mathrm{~nm}$ ). GFP signal was imaged using the DC300 F digital camera system (Leica microsystems).

\section{Detection of mutations in genomic DNA}


Genomic DNA was extracted from the cell mass, the aerial part, or the root using the DNAs-ici!-P kit (Rizo inc. Tsukuba, Japan) or "One-step method" according to the instructions of the KOD FX Neo kit (Toyobo, Osaka, Japan). Target regions were amplified using the KOD -Plus- Neo kit (Toyobo) or KOD FX neo kit (Toyobo). The GFP expression cassette was amplified using the p\#113_f and p\#49_r primer pair (Supplementary Table S1). PCR conditions were as follows: $94^{\circ} \mathrm{C}$ for $2 \mathrm{~min} ; 30-35$ cycles at $98^{\circ} \mathrm{C}$ for 10 $\mathrm{s}, 58^{\circ} \mathrm{C}$ for $30 \mathrm{~s}$, and $68^{\circ} \mathrm{C}$ for $30 \mathrm{~s}$; and final extension at $68^{\circ} \mathrm{C}$ for $7 \mathrm{~min}$. PCR products were sequenced directly using p\#89_r for GFP target \#1 and p\#87_r for GFP target \#2_rev and \#3_rev. For cell lines with multiple indel patterns, PCR products were cloned into PCRIl using the Zero-blunt PCR TOPO kit (Invitrogen, Waltham, Massachusetts). Sequence analysis was performed for each clone. To analyze mutation patterns of $\mathrm{CjCh//targeted} \mathrm{mutagenesis} \mathrm{individuals,} \mathrm{a} \mathrm{fragment} \mathrm{containing} \mathrm{the} \mathrm{target} \mathrm{sequence}$ was amplified using primers p\#468_f and p\#648_r (Supplementary Table S1). PCR conditions were as follows: $94^{\circ} \mathrm{C}$ for $2 \mathrm{~min} ; 40$ cycles at $98^{\circ} \mathrm{C}$ for $10 \mathrm{~s}, 65^{\circ} \mathrm{C}$ for $30 \mathrm{~s}$, and $68^{\circ} \mathrm{C}$ for $20 \mathrm{~s}$; and final extension at $68^{\circ} \mathrm{C}$ for $7 \mathrm{~min}$. PCR products were cloned into pCRIl using the Zero-blunt PCR TOPO kit, and then, sequenced using M13 forward or M13 reverse primers.

\section{Southern blot analysis}

Genomic DNA was isolated from ETs and young needles using Nucleon PhytoPure (Cytiva, Tokyo, Japan). DIG-labeled PCR probes were amplified using p\#89_f and p\#301_r primers for GFP and \#466_f and \#501_r primers for CjChII (Supplementary Table S1), according to the manufacturer's instructions (PCR DIG Probe Synthesis Kit, Roche Diagnostics, Mannheim, Germany). Hybridized probes were detected using anti-Dioxigenin-AP (Roche Diagnostics) on an optical imaging system (Fusion Solo 4M, Vilber Lourmat, Marne la Vallee, France).

\section{Heteroduplex mobility assay}

A 215 bp fragment containing the target sites of CjCh//was amplified using primers p\#468_f and p\#469_r (Supplementary Table S1). PCR amplification was performed under the following conditions: $94^{\circ} \mathrm{C}$ for 2 min; 40 cycles at $98^{\circ} \mathrm{C}$ for $10 \mathrm{~s}, 65^{\circ} \mathrm{C}$ for $30 \mathrm{~s}$, and $68^{\circ} \mathrm{C}$ for $20 \mathrm{~s}$; and final extension at $68^{\circ} \mathrm{C}$ for $7 \mathrm{~min}$. To ensure full heteroduplex formation, a denaturation and re-annealing procedure was performed on the PCR products as follows: $95^{\circ} \mathrm{C}$ for $5 \mathrm{~min} ; 95^{\circ} \mathrm{C}$ to $85^{\circ} \mathrm{C}$, ramping at $-2{ }^{\circ} \mathrm{C} / \mathrm{s}$; and $85^{\circ} \mathrm{C}$ to $25^{\circ} \mathrm{C}$ at -0.1 ${ }^{\circ} \mathrm{C}$. Reannealed products were analyzed using a microchip electrophoresis system (MCE-202 MultiNA, Shimazu, Kyoto, Japan) with the DNA-500 reagent kit (Shimazu).

\section{Statistical analysis}

Pairwise multiple comparison of proportions was performed using Tukey's multiple comparison test conducted in R (https://www.R-project.org/). Significant results were assumed for $P<0.05$. 


\section{Results}

\section{Knock-out of a reporter GFP transgene using the CRISPR/Cas9 system with various U6 promoters}

We tested the efficiency of the CRISPR/Cas9 system in C. japonica. To obtain an optimal promoter for driving the expression of $S p C a s 9$, we screened seven constitutive promoters (Supplementary Information) using the dual luciferase (LUC) transient expression assay. PcUbi was found to be the most active (Supplementary Fig. S2) in the ET of $C$. japonica and considered the optimal promoter for driving $S p C a s 9$ expression. Hereafter, PcUbi was used as the promoter for Cas9 (Fig. 1). Next, to screen for the optimum U6 promoter for gRNA expression, we isolated endogenous U6 promoters in C. japonica using TAIL-PCR ${ }^{33}$ with specific primers corresponding to U6 snRNA consensus sequences and isolated 11 putative U6 promoter fragments (Supplementary Information). Sequence analysis of these fragments revealed that all had an upstream sequence element (USE) and TATA-like box sequence, which are typical characteristics of U6 promoters ${ }^{34}$ (Supplementary Fig. S3). To test U6 promoter activity, the GFPexpressing ET line N4, which contains a single-copy transgene (Supplementary Fig. S1), was obtained and used for the CRISPR/Cas9-mediated GFP knock-out experiment. We selected target sites that have been reported ${ }^{35-37}$ (Table 1). pZK_FFCas9 vectors containing the gRNA of GFP target \#1 driven by 13 U6 promoters were introduced into N4, and 17-138 transgenic ET lines were obtained for each vector (Table 2) by screening in a medium with $25 \mathrm{mg} / \mathrm{L}$ kanamycin. Loss of GFP fluorescence lines were observed in 1-39 lines for each transformed line (Table 2). GFP fluorescence was lost in some lines completely, with others exhibiting GFP fluorescence in some areas of the cell mass (Fig. 2). By contrast, all transgenic lines with the Cas9 expression cassette alone (without the gRNA expression cassette) exhibited GFP fluorescence (Fig. 2, Table 2, Supplementary Fig. S4). GFP knock-out efficiency and number of lines isolated varied within U6 promoters (range, 3.1-37.9\%) (Table 2). Target-dependent knock-out efficiency using the Arabidopsis U6 promoter (Table 3) was 41.4\% and 31.2\% using target \#2_rev and target \#3_rev, respectively, which were higher than that for target \#1 (25.0\%). Mutation patterns in each line were confirmed through sequencing (Fig. 3, Supplementary Fig. S5). Frequent modifications in all targets were single-nucleotide insertions. Additionally, single-nucleotide deletions were often observed in target \#2_rev and target \#3_rev. By contrast, large deletions, such as 15 bp deletions in target \#1 and 33 bp deletion in target \#3_rev, were the most frequent modifications. From the point onward, CjU6_\#2 promoter was used for gRNA expression in $C$. japonica. 
Table 2

Effect of U6 promoters on the efficiency of targeted mutagenesis of the GFP transgene

\begin{tabular}{lllll} 
Target & $\begin{array}{l}\text { U6 } \\
\text { promoter }\end{array}$ & $\begin{array}{l}\text { No. of transformed lines } \\
(A)\end{array}$ & $\begin{array}{l}\text { No. of GFP knock out lines } \\
(\mathbf{B})\end{array}$ & $\begin{array}{l}\text { Efficiency } \\
(\%)\end{array}$ \\
\hline$-\S$ & 63 & 0 & 0 \\
\hline CjU6_\#1 & 138 & 39 & $28.3^{\mathrm{a}}$ \\
\hline CjU6_\#2 & 134 & 33 & $24.6^{\mathrm{a}}$ \\
\hline CjU6_\#3 & 98 & 3 & $3.1^{\mathrm{c}}$ \\
\hline CjU6_\#4 & 29 & 11 & $37.9^{\mathrm{a}}$ \\
\hline CjU6_\#5 & 72 & 15 & $20.8^{\mathrm{ab}}$ \\
\hline CjU6_\#6 & 39 & 4 & $10.3^{\mathrm{abc}}$ \\
\hline CjU6_\#7 & 55 & 9 & $16.4^{\mathrm{abc}}$ \\
\hline CjU6_\#8 & 20 & 1 & $5.0^{\mathrm{abc}}$ \\
\hline CjU6_\#9 & 17 & 3 & $17.6^{\mathrm{abc}}$ \\
\hline CjU6_\#10 & 116 & 18 & $15.6^{\mathrm{abc}}$ \\
\hline CjU6_\#11 & 60 & 2 & $3.3^{\mathrm{bc}}$ \\
\hline OsU6 & 50 & 12 & $24.0^{\mathrm{ab}}$ \\
\hline AtU6-26 & 136 & 34 & $25.0^{\mathrm{a}}$ \\
\hline
\end{tabular}

*The number of calluses that completely lost GFP fluorescence or had GFP fluorescence in some parts of the cell mass was counted after $87 \mathrm{~d}$ of selective cultivation. ${ }^{\dagger}(\mathrm{B} / \mathrm{A}) \times 100$. ${ }^{\ddagger}$ Pairwise multiple comparison of proportions was performed using Tukey's multiple comparison test. Proportions with significant difference were labeled with different letters $(P<0.05)$. SpZK_FFCas9 vector without gRNA expression cassette-introducing N4 transgenic lines (Negative control). 
Table 3

Effect of target sequence on the efficiency of targeted mutagenesis of the GFP transgene

\begin{tabular}{|c|c|c|c|c|c|}
\hline Target $^{*}$ & $\begin{array}{l}\text { No. of } \\
\text { transformed } \\
\text { lines }(A)\end{array}$ & $\begin{array}{l}\text { No. of GFP- } \\
\text { knock-out } \\
\text { lines }^{\dagger}(B)\end{array}$ & $\begin{array}{l}\text { Mutation } \\
\text { frequency } \\
(\%)^{\ddagger}\end{array}$ & $\begin{array}{l}\text { No. of lines having } \\
\text { multiple mutation } \\
\text { patterns } \S \text { (C) }\end{array}$ & $\begin{array}{l}\text { Mosaicism } \\
\text { frequency } \\
\text { (\%) }\end{array}$ \\
\hline \#1 & $136 *$ & $34^{*}$ & $25.0 *, b$ & 15 & $44.1^{c}$ \\
\hline \#2_rev & 70 & 29 & $41.4^{\mathrm{a}}$ & 17 & $58.6^{c}$ \\
\hline \#3_rev & 77 & 24 & $31.2^{\mathrm{ab}}$ & 15 & $62.5^{\mathrm{c}}$ \\
\hline \multicolumn{6}{|c|}{$\begin{array}{l}\text { ET; embryogenic tissue. }{ }^{*} \text { pZK_FFCas9 vectors with gRNA expression cassettes driven by AtU6-26 } \\
\text { promoter were transformed into ET. }{ }^{\dagger} \text { The number of ET lines was counted after } 87 \mathrm{~d} \text { of selective } \\
\text { cultivation. }{ }^{\ddagger}(\mathrm{B} / \mathrm{A}) \times 100 \text {. Pairwise multiple comparison of proportions was performed using Tukey's } \\
\text { multiple comparison test. Proportions with significant difference were labeled with different letters (P } \\
<0.05) . \text { SThe number of ET lines having multiple mutation patterns was counted. }^{9}(\mathrm{C} / \mathrm{B}) \times 100 \text {. } \\
\text { Pairwise multiple comparison of proportions was performed using Tukey's multiple comparison test. } \\
\text { Proportions with a significant difference were labeled with different letters }(P<0.05) \text {. *Data from } \\
\text { Table } 1 \text {. }\end{array}$} \\
\hline
\end{tabular}

\section{Investigation of chimerism in the regenerated T0 plantlet}

Loss of GFP fluorescence was frequently partial in many transformed lines (Fig. 2) and ranged from $44.1-62.5 \%$ depending on the target sequence (Table 3 ). This is because mutations occur independently in a single cell; therefore, a variety of mutations can be obtained from a single transgenic line ${ }^{38}$. If the plantlet was derived from genomes of edited and non-edited cells, the plantlet may exhibit chimerism or mosaicism, such that the plantlet will have functional GFP in one portion and mutated non-functional GFP in the other portion. During the development of somatic cotyledonary embryos, GFP fluorescence clearly separated into positive or negative; based on our observations, no cotyledon exhibited chimeric GFP fluorescence (Fig. $4 \mathrm{a}-\mathrm{j}$ ). In a green germinating somatic embryo with active GFP, GFP fluorescence could hardly be observed due to strong chlorophyll autofluorescence; conversely, GFP fluorescence was observed in the callus at the base of the embryo (Fig. 4d, i). To investigate mosaicism in cotyledonary somatic embryos, sequencing analysis was performed using 8-30 cotyledonary embryos (139 from 6 lines). One cotyledonary embryo from line \#47 - 2 had two modification patterns in one individual somatic embryo. However, in others, mutation patterns were clearly separated in individual cotyledonary embryos (Fig. 4k). Next, the stability of mutation patterns was analyzed in 10 plantlets regenerated through somatic embryogenesis in each of 4 lines, namely \#42-2,\#18, \#31-2, and \#11, by analyzing the sequence from multiple needle and root segments (Supplementary Fig. S6a). All plantlets regenerated from \#31 - 2 and \#11 lines had only one mutation pattern in all tissue segments. By contrast, in lines $\# 42$ - 2 and \#18, plantlets with additional mutation(s) in root segments were observed (Supplementary Fig. S6b). No apparent mosaicism was detected in these tissues. 


\section{Targeted mutagenesis of an endogenous gene using the CRISPR/Cas9 system}

We modified an endogenous $C$. japonica gene using CRISPR/Cas9. We selected $C h l l$, which is required for chlorophyll biosynthesis ${ }^{39}$. Chll mutants exhibit an albino phenotype ${ }^{40}$. A 2,152 bp fragment corresponding to CjCh// was cloned (Accession no. LC603656) (Fig. 5a). Southern blot analysis suggested that CjCh/l is a single gene (Fig. 5b). To introduce mutations in CjChll, we designed two targeting sequences in exon 3 of $C j C h l l$, named t 1 and t2, respectively (Fig. 5a). Three binary vectors for disrupting CjCh// were constructed using the pCRG-SpCas9 vector (Fig. 5c, Supplementary Information). Transgenic lines were selected in a kanamycin-containing medium. Then, putative genome-edited lines were screened using the heteroduplex mobility assay (HMA) (Fig. 6a), leading to the selection of 7, 4, and 2 lines for $\mathrm{t} 1$, $\mathrm{t} 2$, and $\mathrm{t} 1+\mathrm{t} 2$, respectively (Table 4 ). Transgenic plantlets were induced through mature somatic embryos; some lines failed to produce somatic embryos, and regenerated shoots with visible phenotypes, namely albino, pale green, and green (Fig. 6b-g). Albino transformants (Fig. 6b-e) grew very slowly. Pale green transformant (Fig. 6f) grew slower than wild-type. A green transformant (Fig. 6g) grew similar to wild-type (Fig. 6h). To verify the modification of sequences at target sites in transformants induced by the CRISPR/Cas9 system, sequences around the target site were analyzed from genomic DNA isolated from aerial part of transformants. PCR products were cloned into a cloning vector and 15-32 clones were analyzed for each transformant. Various mutations were detected at target sites in these lines (Fig. 6i), indicating that targeted mutagenesis of CjCh// occurred. Moreover, CjCh// was completely disrupted in albino transformants. By contrast, a point mutation was observed in 8 of 15 clones in the pale green transformant. In the green transformant ( $\left.1+t 2 \_\# 6-2 \_3\right), 1$ of 16 clones had a deletion in the target site. Taken together, our results indicate that the CRISPR/Cas 9 system induced targeted mutagenesis through double-strand breaks in an endogenous gene of $C$. japonica.

Table 4

Summary of endogenous Cryptomeria japonica magnesium chelatase subunit I (CjCh/) modification using the CRISPR/Cas9 system

\begin{tabular}{|llll|}
\hline Target $^{*}$ & No. of transformed lines (A) & No. of HMA positive lines (B) & Mutation frequency (\%) \\
\hline $\mathrm{t} 1$ & 20 & 7 & $35^{\mathrm{a}}$ \\
$\mathrm{t} 2$ & 17 & 4 & $23.5^{\mathrm{a}}$ \\
$\mathrm{t} 1+\mathrm{t} 2$ & 7 & 2 & $28.6^{\mathrm{a}}$
\end{tabular}

*pCRG-SpCas9 vectors with gRNA expression cassettes driven by CjU6_\#2 promoter were transformed into embryogenic tissue. ${ }^{\dagger}(B / A) \times 100$. Pairwise multiple comparison of proportions was performed using Tukey's multiple comparison test. Proportions with significant difference were labeled with different letters $(P<0.05)$.

\section{Discussion}


Here we report targeted mutagenesis through the CRISPR/Cas9 system in $C$. japonica, which is a first in coniferous trees species to the best of our knowledge. Genetic transformation of conifers is expected to be a powerful tool for modifying their characteristics in a short time. This technology has been used for producing herbicide-tolerant and insect-resistant crops, such as corn, soybean, and cotton ${ }^{41}$. However, these are mostly annual crops, and little research has been done on how transgenes behave long-term (> 10 years) in perennial plants, such as trees. In addition, unlike crop cultivation, trees grow in forests, where human management is a challenge. Studies have also raised concerns about transgene instability ${ }^{42}$. Genome-edited plants with no transgenes are attractive for perennial tree species in terms of public acceptance. Inducing gibberellin-mediated flowering in $C$. japonica is easy. Among conifers, obtaining null segregants through mating in a relatively short time is possible in $C$. japonica, because it is easy to induce earlier flowering in even one-year-old seedling plants by gibberellin treatment ${ }^{43}$. Moreover, C. japonica, which has a stable culture, transformation system, and genome editing system, could be a model for coniferous tree species.

During vector development, AtHSP and NOS terminators in tandem were found to be important for the stable expression of exogenous proteins. The pZmUbi-GFP-Dt vector-introducing lines maintained strong GFP fluorescence after 5 years of subculture. In this study, we also surveyed the activity of U6 promoters isolated from $C$. japonica, in addition to the commonly used ones from A. thaliana and Oryza sativa, for improving mutation efficiency. In some plant species, such as soybean and cotton, mutation efficiency increased when their intrinsic U6 promoters were used instead of the Arabidopsis U6 promoter ${ }^{44,45}$. However, contrary to expectations, the efficiency of genome editing using the U6 promoters of $C$. japonica was not significantly different from that with the U6 promoters of $A$. thaliana or 0 . sativa (Table 2). Whether this was due to the activity of the U6 promoter or the expression level of Cas 9 is unknown. The terminator used for the Cas9 expression cassette in pZK_FFCas9 (Fig. 1b) might be unsuitable for Cas9 expression, compared with the tandem terminator unit of AtHSPt and NOSt in the pCRG-SpCas 9 vector (Fig. 5c). Whether our results apply to all conifers warrants further study using other target tree species.

In the GFP-expressing line (N4), mutation frequency with GFP target \#2_rev was 41.4\% (Table 3). By contrast, Jacob et al. ${ }^{37}$ detected indels in more than $70 \%$ transgenic lines when targeting the same GFP region in a genome editing study using GFP-expressing hairy roots of soybeans. These results may indicate that the amount of Cas 9 in $C$. japonica was lower than that in soybean. Short indel patterns were observed in a large number of transgenic lines (Fig. 3, Supplementary Fig. S5), which is consistent with the results of others ${ }^{21,46}$. In addition, some transgenic lines had large deletions, such as 15 and 33 bp in GFP target \#1 and target \#3_rev, respectively (Fig. 3 and Supplementary Fig. S5). This deletion may be a microhomology-associated deletion ${ }^{47}$, that is, microhomologies, such as "TCGA" and "GCTG," between the target sequence in GFP (Supplementary Fig. S5). Chimerism or mosaicism is an important concern in the application of genome editing in plant species; especially generation intervals are counted in years. Transgenic lines with mottled GFP knock-out regions were used to investigate chimerism in induced somatic embryos; however, more than $99 \%$ of embryos had a single modification pattern (Fig. 4k). The results support the hypothesis that the somatic embryo culture system originated from single cells ${ }^{48}$. 
However, we also observed new modifications in the root tissue as the individual grew larger (Supplementary Fig. S6b). This result suggests that Cas9-mediated DNA cleavage is ongoing. Furthermore, the promoter activity of PcUbi is expected to be higher in the root tissue than in ET and needle tissue. These results also imply that if mutated embryos could be isolated, the chimerism would be eliminated. A difference in editing efficiency depending on the target sequence was observed (Table 3). Doench et al. ${ }^{49}$ developed a tool to predict gRNA activity; however, no correlation between the score value and actual cutting efficiency was observed. No effective tool is available for predicting gRNA cleavage efficiency in plants ${ }^{50}$.

We succeeded in performing targeted mutagenesis of endogenous CjCh/I (Fig. 6b-g). By contrast, a mutation was observed in one of 16 clones from the green transformant line t1 + t2_\#6-2_3 (Fig. 6g, i). This result indicates that continuous DNA cleavage by Cas 9 and prolonged cultivation can lead to the modification of target regions. Further improvements in the CRISPR/Cas 9 expression vector are required for more efficient mutagenesis. Codon optimization of Cas9 dramatically increases the efficiency of obtaining transformants with mutations in both alleles (Nanasato et al., in preparation). We are conducting genome editing studies using the improved vector and producing genome-edited male-sterile lines. The use of genome editing technology in confer species will directly contribute to the analysis of gene function in conifers, which has been difficult to analysis so far.

To achieve the full potential of genome editing technologies in conifer species, detailed genomic information needs to be available. In addition, an efficient technique for producing transgene-free individuals, through methods other than crossing, must be developed for practical use. In many crops, crossing has been used to obtain transgene-free individuals. However, crossing in conifers takes years. Furthermore, conifer species are not autogamous, and during crossing with another individual, useful parental traits are lost in the offspring. Direct delivery of the Cas9 and sgRNA complex into plant cells is an attractive solution ${ }^{51,52}$. The cell wall is a major obstacle to the direct delivery of proteins. Yanagawa et

al. ${ }^{53}$ used a multi-gas plasma jet to deliver proteins into tobacco leaf. We have discovered a cellpenetrating peptide, which delivers proteins directly into $C$. japonica cells (Tanaka, Nanasato, et al., in submission). We believe that the integration of the efficient genome editing system we report here and direct protein delivery techniques will accelerate basic research and molecular breeding in conifers.

\section{Declarations}

\section{Acknowledgements}

We are grateful to Dr. F. Fauser (Sangamo Therapeutics, Inc.), Dr S. Schiml and Professor H. Puchta at University of Karlsruhe Institute of Technology for providing the pDe-CAS9 vector and PcUbi promoter. We thank Professor M. Sakuta of Ochanomizu University for a gift of 35S::LUC and 35S::RUC vectors. We also thank Dr. H. Saika, Dr. S. Toki of NARO, and Professor K. Osakabe and Professor Y. Osakabe of Tokushima University for critical advice. Ms. T. Okuyama, Ms. F. Yamakawa, Ms. K. Ohbu, and J. Orita are 
warmly acknowledged for their experimental support. This work was supported by JSPS KAKENHI Grant Numbers JP16H04942 and JP17K07854.

\section{Author Contributions}

Y.N. is responsible for the overall design of the research. Y.N., K.K., and T.T. conceived, supervised, and designed the experiments; Y.N., M.M., N.F., M.E., Y.O., and M.N. performed experiments; Y.N. wrote the paper. All authors have discussed the results and agreed with the content of the submitted paper.

\section{Conflict of Interest}

The authors declare that they have no conflict of interest.

\section{References}

1. Tsumura, Y. et al. Effects of the last glacial period on genetic diversity and genetic differentiation in Cryptomeria japonica in East Asia. Tree Genet. Genomes 16, 19 (2020).

2. Ohba, K. Clonal Forestry with Sugi (Cryptomeria japonica). in Clonal Forestry II (eds. Ahuja, M. \& Libby, W.) 66-90 (Springer Berlin Heidelberg, 1993). doi:10.1007/978-3-642-84813-1_4

3. Kondo, T. \& Kuramoto, N. Cryptomeria japonica. in Forest Trees (ed. Kole, C.) 211-221 (Springer Berlin Heidelberg New York, 2007).

4. Forest Agency, Japan. State of Japan's Forests and Forest Management -3rd Country Report of Japan to the Montreal Process-. http://www.rinya.maff.go.jp/j/kaigai/pdf/countryreport-1.pdf/ (2019).

5. Yamada, T., Saito, H. \& Fujieda, S. Present state of Japanese cedar pollinosis: The national affliction. J. Allergy Clin. Immunol. 133, 632-639.e5 (2014).

6. Tsubomura, M., Kurita, M. \& Watanabe, A. Determination of male strobilus developmental stages by cytological and gene expression analyses in Japanese cedar (Cryptomeria japonica). Tree Physiol. 36, 653-666 (2016).

7. Futamura, N., Igasaki, T., Saito, M., Taira, H. \& Shinohara, K. Comparison of fertile and sterile male gametogenesis in Cryptomeria japonica D. Don. Tree Genet. Genomes 15, (2019).

8. Hasegawa, Y. et al. Fine mapping of the male-sterile genes (MS1, MS2, MS3, and MS4) and development of SNP markers for marker-assisted selection in Japanese cedar (Cryptomeria japonica D. Don). PLoS One 13, e0206695 (2018).

9. Hasegawa, Y. et al. Identification and genetic diversity analysis of a male-sterile gene (MS1) in Japanese cedar (Cryptomeria japonica D. Don). Sci. Rep. 11, 1496 (2021).

10. Mishima, K. et al. Identification of novel putative causative genes and genetic marker for male sterility in Japanese cedar (Cryptomeria japonica D.Don). BMC Genomics 19, 1-16 (2018). 
11. Maruyama, T. E., Ueno, S., Hirayama, S., Kaneeda, T. \& Moriguchi, Y. Somatic embryogenesis and plant regeneration from sugi (Japanese cedar, Cryptomeria japonica D. Don, Cupressaceae) seed families by marker assisted selection for the male sterility allele $m s 1$. Plants 9,1029 (2020).

12. Jinek, M. et al. A programmable dual-RNA - Guided DNA endonuclease in adaptive bacterial immunity. Science 337, 816-822 (2012).

13. Shan, S., Soltis, P. S., Soltis, D. E. \& Yang, B. Considerations in adapting CRISPR/Cas9 in nongenetic model plant systems. Appl. Plant Sci. 8, 1-17 (2020).

14. Nishimasu, H. et al. Engineered CRISPR-Cas9 nuclease with expanded targeting space. Science $\mathbf{3 6 1}$, 1259-1262 (2018).

15. Walton, R. T., Christie, K. A., Whittaker, M. N. \& Kleinstiver, B. P. Unconstrained genome targeting with near-PAMless engineered CRISPR-Cas9 variants. Science 368, 290-296 (2020).

16. Ran, F. A. et al. In vivo genome editing using Staphylococcus aureus Cas9. Nature 520, 186-190 (2015).

17. Pausch, P. et al. CRISPR-Cas $\Phi$ from huge phages is a hypercompact genome editor. Science $\mathbf{3 6 9}$, 333-337 (2020).

18. Nishida, K. et al. Targeted nucleotide editing using hybrid prokaryotic and vertebrate adaptive immune systems. Science 353, 1248 (2016).

19. Gaudelli, N. M. et al. Programmable base editing of $A \cdot T$ to $G \cdot C$ in genomic DNA without DNA cleavage. Nature 551, 464-471 (2017).

20. Anzalone, A. V. et al. Search-and-replace genome editing without double-strand breaks or donor DNA. Nature 576, 149-157 (2019).

21. Fan, D. et al. Efficient CRISPR/Cas9-mediated targeted mutagenesis in Populus in the first generation. Sci. Rep. 5, 12217 (2015).

22. Zhou, X., Jacobs, T. B., Xue, L.-J., Harding, S. A. \& Tsai, C.-J. Exploiting SNPs for biallelic CRISPR mutations in the outcrossing woody perennial Populus reveals 4-coumarate: CoA ligase specificity and redundancy. New Phytol. 208, 298-301 (2015).

23. Taniguchi, T., Ohmiya, Y., Kurita, M., Tsubomura, M. \& Kondo, T. Regeneration of transgenic Cryptomeria japonica D. Don after Agrobacterium tumefaciens-mediated transformation of embryogenic tissue. Plant Cell Rep. 27, 1461-1466 (2008).

24. Konagaya, K. I., Nanasato, Y. \& Taniguchi, T. A protocol for Agrobacterium-mediated transformation of Japanese Cedar, Sugi (Cryptomeria japonica D. Don) using embryogenic tissue explants. Plant Biotechnol. 37, 147-156 (2020).

25. Nagaya, S., Kawamura, K., Shinmyo, A. \& Kato, K. The HSP terminator of Arabidopsis thaliana increases gene expression in plant cells. Plant Cell Physiol. 51, 328-332 (2010).

26. Mikami, M., Toki, S. \& Endo, M. Parameters affecting frequency of CRISPR/Cas 9 mediated targeted mutagenesis in rice. Plant Cell Rep. 34, 1807-1815 (2015). 
27. Fauser, F., Schiml, S. \& Puchta, H. Both CRISPR/Cas-based nucleases and nickases can be used efficiently for genome engineering in Arabidopsis thaliana. Plant J. 79, 348-59 (2014).

28. Kuroda, M., Kimizu, M. \& Mikami, C. A simple set of plasmids for the production of transgenic plants. Biosci. Biotechnol. Biochem. 74, 2348-2351 (2010).

29. Endo, M., Nishizawa-Yokoi, A. \& Toki, S. Targeted Mutagenesis in Rice Using TALENs and the CRISPR/Cas9 System. in Chromosome and Genomic Engineering in Plants: Methods and Protocols (ed. Murata, M.) 1469, 123-135 (Springer Science+Business Media, LLC, NY, 2016).

30. Kawalleck, P., Somssich, I. E., Feldbrügge, M., Hahlbrock, K. \& Weisshaar, B. Polyubiquitin gene expression and structural properties of the ubi4-2 gene in Petroselinum crispum. Plant Mol. Biol. 21, 673-684 (1993).

31. Hyun, Y. et al. Site-directed mutagenesis in Arabidopsis thaliana using dividing tissue-targeted RGEN of the CRISPR/Cas system to generate heritable null alleles. Planta 241, 271-284 (2014).

32. Konagaya, K. I., Kurita, M. \& Taniguchi, T. High-efficiency Agrobacterium-mediated transformation of Cryptomeria japonica D. Don by co-cultivation on filter paper wicks followed by meropenem treatment to eliminate Agrobacterium. Plant Biotechnol. 30, 523-528 (2013).

33. Liu, Y.-G., Mitsukawa, N., Oosumi, T. \& Whittier, R. F. Efficient isolation and mapping of Arabidopsis thaliana T-DNA insert junctions by thermal asymmetric interlaced PCR. Plant Journal 8, 457-463 (1995).

34. Waibel, F. \& Filipowicz, W. U6 snRNA genes of Arabidopsis are transcribed by RNA polymerase III but contain the same two upstream promoter elements as RNA polymerase II-transcribed U-snRNA genes. Nucleic Acids Res. 18, 3451-3458 (1990).

35. Fu, Y. et al. High-frequency off-target mutagenesis induced by CRISPR-Cas nucleases in human cells. Nat. Biotechnol. 31, 822-826 (2013).

36. Mali, P. et al. RNA-Guided Human Genome Engineering via Cas9. Science 339, 823-826 (2013).

37. Jacobs, T. B., Lafayette, P. R., Schmitz, R. J. \& Parrott, W. A. Targeted genome modifications in soybean with CRISPR/Cas9. BMC Biotechnol. 15, 1-10 (2015).

38. Sugano, S. S. et al. Genome editing in the mushroom-forming basidiomycete Coprinopsis cinerea, optimized by a high-throughput transformation system. Sci. Rep. 7, 1-9 (2017).

39. Kruse, E., Mock, H. \& Grimm, B. Isolation and characterisation of tobacco (Nicotiana tabacum) cDNA clones encoding proteins involved in magnesium chelation into protoporphyrin IX. Plant Mol. Biol. 35, 1053-1056 (1997).

40. Koncz, C. et al. Isolation of a gene encoding a novel chloroplast protein by T-DNA tagging in Arabidopsis thaliana. EMBO J. 9, 1337-1346 (1990).

41. Kumar, K. et al. Genetically modified crops: current status and future prospects. Planta 251, 1-27 (2020).

42. Hoenicka, H. \& Fladung, M. Genome Instability in Woody Plants Derived from Genetic Engineering. in Tree Transgenesis (eds. Fladung, M. \& Ewald, D.) 301-321 (Springer Berlin Heidelberg, 2006). 
doi:10.1007/3-540-32199-3_14

43. Hashizume, $\mathrm{H}$. The effect of gibberellin upon flower formation in Cryptomeria japonica. J. Japanese For. Soc. 41, 375-381 (1959).

44. Long, L. et al. Optimization of CRISPR/Cas9 genome editing in cotton by improved sgRNA expression. Plant Methods 14, 1-9 (2018).

45. Sun, X. et al. Targeted mutagenesis in soybean using the CRISPR-Cas9 system. Sci. Rep. 5, 10342 (2015).

46. Feng, Z. et al. Multigeneration analysis reveals the inheritance, specificity, and patterns of CRISPR/Cas-induced gene modifications in Arabidopsis. Proc. Natl. Acad. Sci. U. S. A. 111, 4632-7 (2014).

47. Bae, S., Kweon, J., Kim, H. S. \& Kim, J. S. Microhomology-based choice of Cas9 nuclease target sites. Nat. Methods 11, 705-706 (2014).

48. Nagmani, R., Becwar, M. R. \& Wann, S. R. Single-cell origin and development of somatic embryos in Picea abies (L.) Karst. (Norway spruce) and P. glauca (Moench) Voss (white spruce). Plant Cell Rep. 6, 157-159 (1987).

49. Doench, J. G. et al. Rational design of highly active sgRNAs for CRISPR-Cas9-mediated gene inactivation. Nat. Biotechnol. 32, 1262-7 (2014).

50. Naim, F. et al. Are the current gRNA ranking prediction algorithms useful for genome editing in plants? PLoS One 15, 1-12 (2020).

51. Woo, J. W. et al. DNA-free genome editing in plants with preassembled CRISPR-Cas 9 ribonucleoproteins. Nat. Biotechnol. 33, 1162-1164 (2015).

52. Svitashev, S., Schwartz, C., Lenderts, B., Young, J. K. \& Mark Cigan, A. Genome editing in maize directed by CRISPR-Cas9 ribonucleoprotein complexes. Nat. Commun. 7, 13274 (2016).

53. Yanagawa, Y. et al. Direct protein introduction into plant cells using a multi-gas plasma jet. PLoS One 12, e0171942 (2017).

\section{Figures}



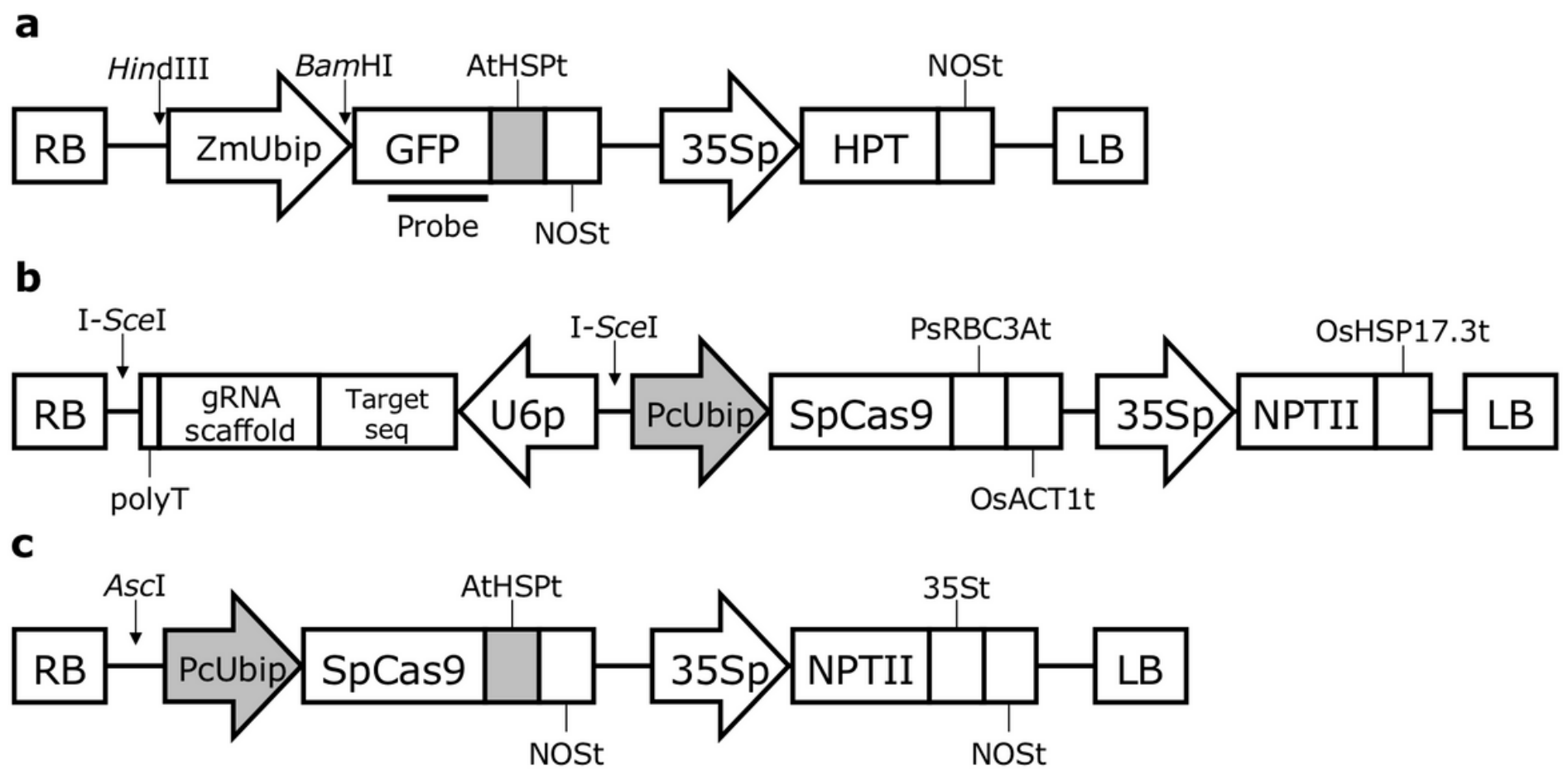

Figure 1

Schematic diagram of the T-DNA region of GFP expression vector (pZmUbi-GFP-Dt, (a)), CRISPR/Cas9 expression vector (pZK_FFCas9 vector with a gRNA expression cassette, (b)), and modified CRISPR/Cas9 expression vector (pCRG-SpCas9, (c)). HindIII and BamHI sites and the DNA fragment used as the probe for Southern blot analysis are indicated in (a). RB, right border; ZmUbip, ubiquitin promoter from Zea mays, AtHSPt, heat shock protein terminator from Arabidopsis thaliana; NOSt, nopaline synthase terminator; $35 \mathrm{Sp}$, cauliflower mosaic virus $35 \mathrm{~S}$ promoter; HPT, hygromycin phosphotransferase; LB, left border; U6p, U6 promoter; PcUbip, ubiquitin promoter from Petroselinum crispum; SpCas9, Cas9 from Streptococcus pyogenes (codon-optimized for A. thaliana); PsRBC3At, RBC3A terminator from Pisum sativum; OsACT1t, actin-1 terminator from Oryza sativa; NPTII, neomycin phosphotransferase; OsHSP17.3t, heat shock protein 17.3 terminator from 0. sativa. 


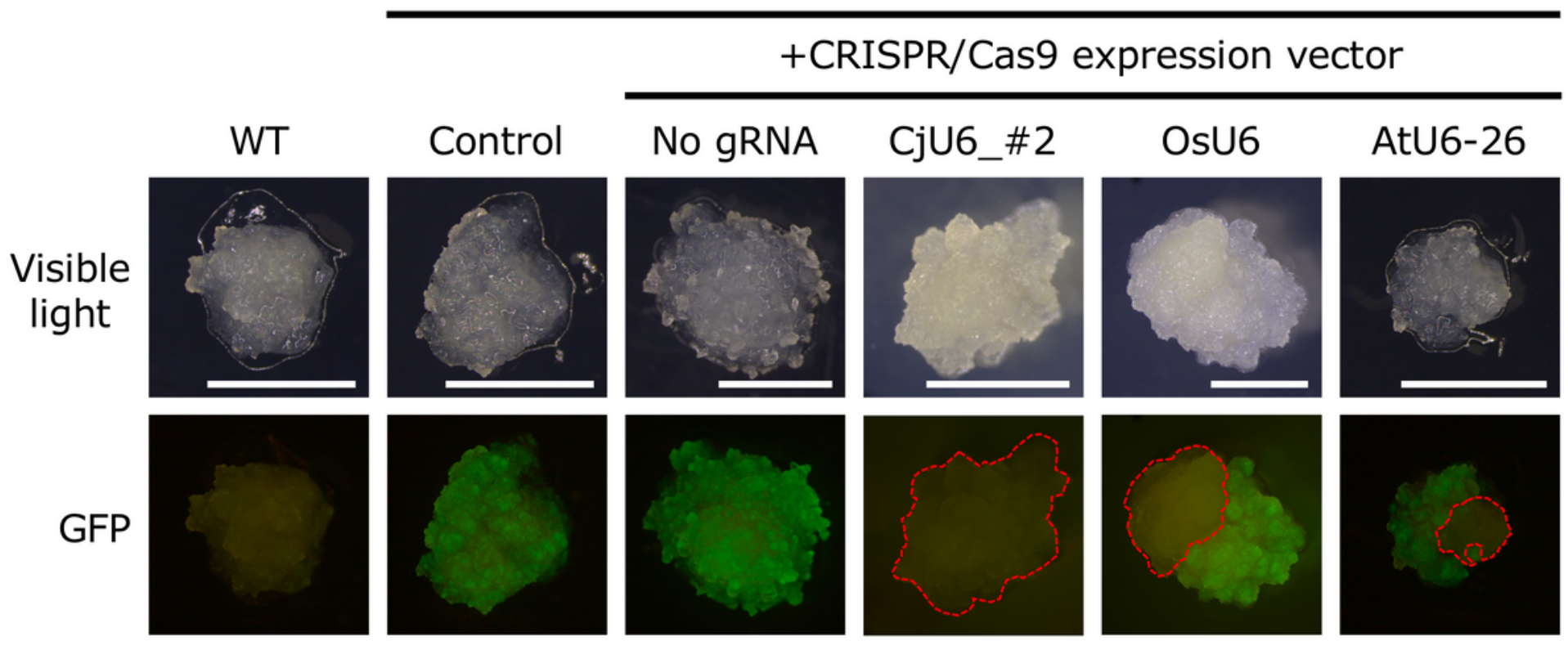

Figure 2

Targeted mutagenesis of GFP in Cryptomeria japonica embryogenic tissue (ET). WT, wild-type cell line \#13-8-12; +GFP, GFP-overexpressing line N4; +CRISPR/Cas9 expression vector, transgenic N4 lines introducing pZF_FFCas9-derived GFP-targeting vectors; No gRNA, ET line \#48 introducing pZK_FF without the gRNA expression cassette; CjU6_\#2, ET line \#50 introducing pZK_FF with the CjU6_\#2-driven GFP target 1 expression cassette vector; OsU6, ET line \#27 introducing pZK_FF with the OsU6-driven GFP target 1 expression cassette vector; and AtU6-26, ET line \#70 introducing pZK_FF with the AtU6-26-driven GFP target 1 expression cassette vector. Area enclosed by red dots indicates cells without GFP fluorescence. Bars, $5 \mathrm{~mm}$. 
a
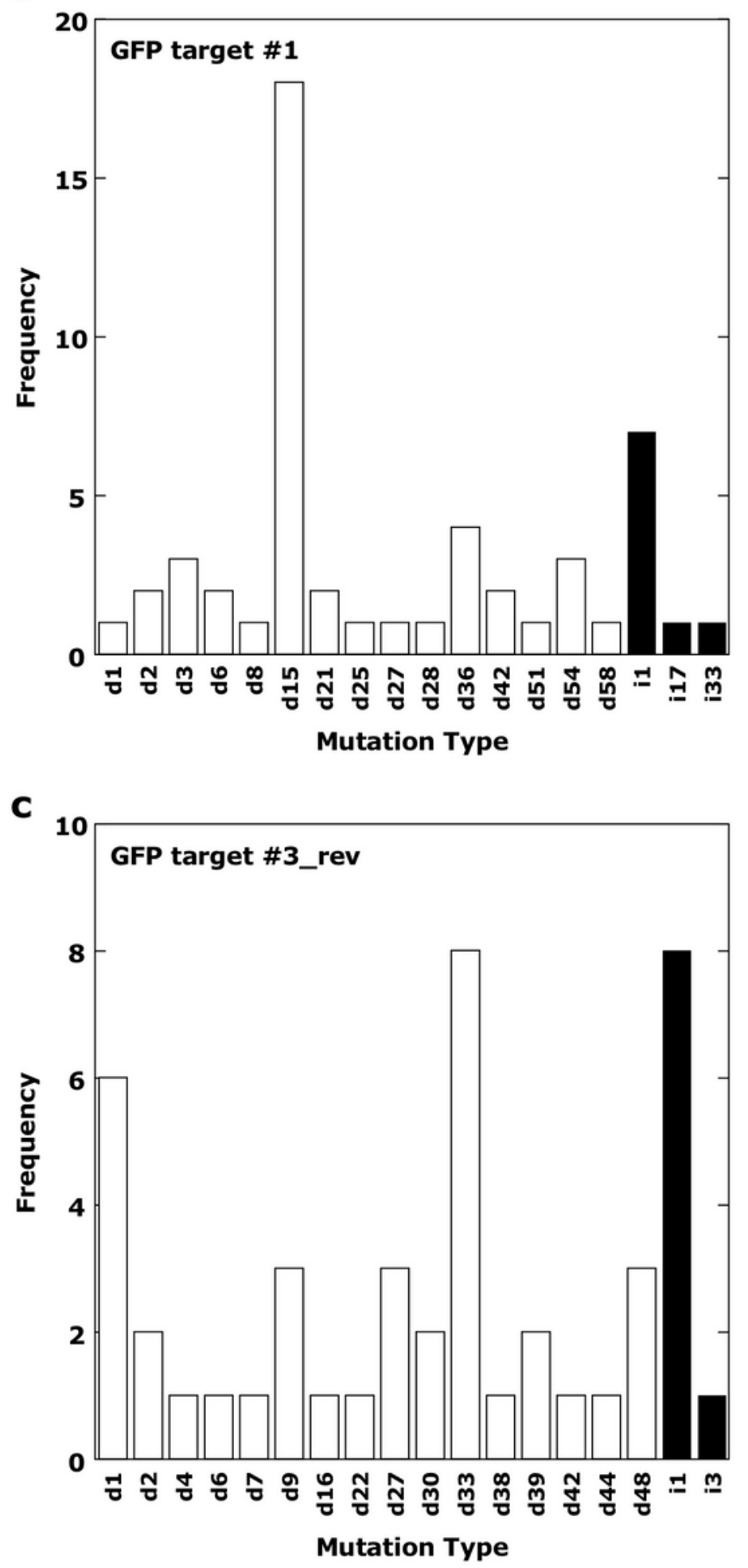

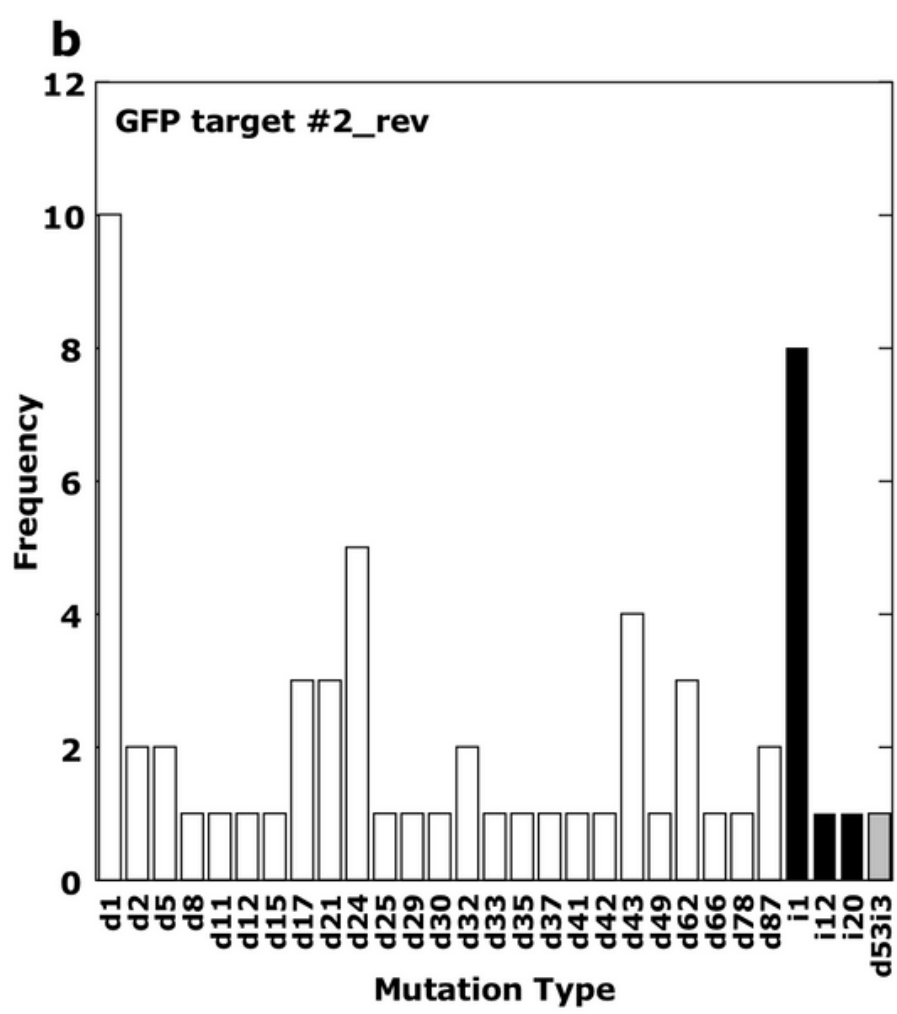

Group

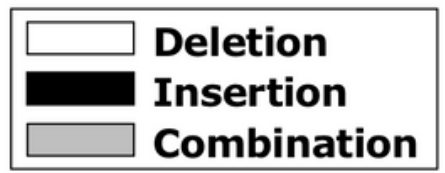

\section{Figure 3}

CRISPR/Cas9-induced target-dependent knock-out mutation type and length. pZK_FFCas9 vectors containing the gRNA of GFP target \#1 (a), \#2_rev (b) and \#3_rev (c) driven by AtU6-26 promoters were introduced into the GFP-expressing embryogenic tissue line N4, respectively. $\mathrm{d \#}$ and i\# denote the number of bp deleted and inserted at the target site, respectively. Group: deletion (white bar), mutation pattern 
containing only deletions; insertion (black bar), mutation pattern containing only insertions; combination (gray bar), mutation pattern containing both deletions and insertions.
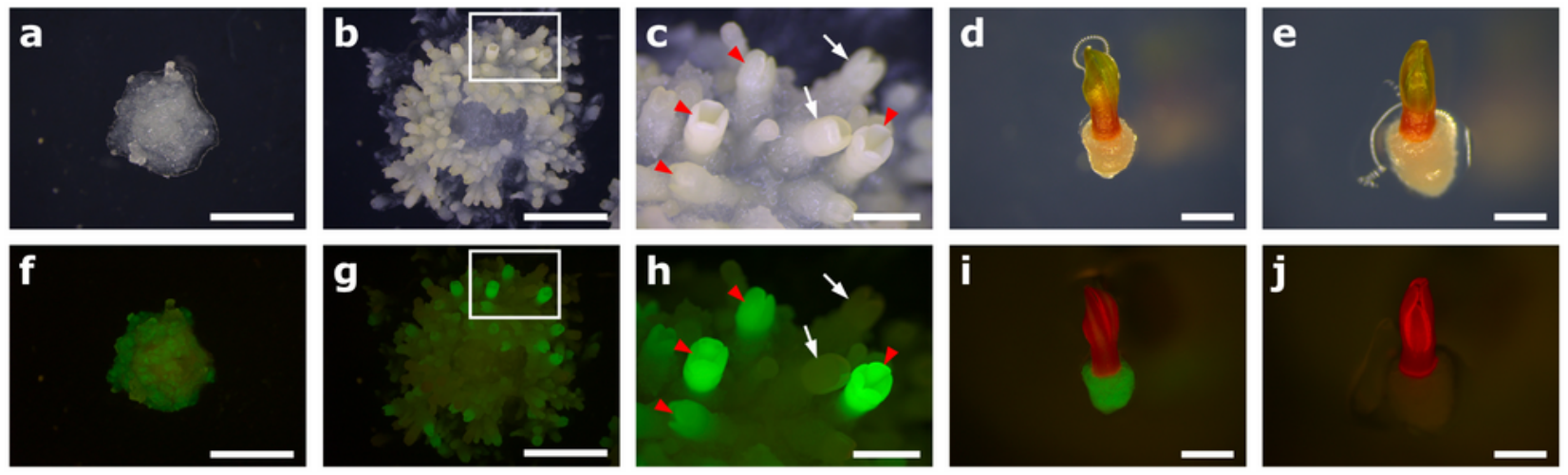

\begin{tabular}{|c|c|c|c|c|}
\hline Line & Sequence of target site $\left(5^{\prime}-3^{\prime}\right)$ & Mutation & $\begin{array}{l}\text { No. of regenerated } \\
\text { somatic embryos }\end{array}$ & $\begin{array}{l}\text { Efficiency of } \\
\text { mutation* }\end{array}$ \\
\hline GFP & AGGGCGACACCCTGGTGAACCGCATCGAGCT GAAGGGCATCGACT & - & - & \\
\hline \multirow[t]{3}{*}{$\# 42-2$} & AGGGCGACACCCTGGTGAACCGCATCGAGCTGAAGGGCATCGACT & - & 21 & $6 / 27$ \\
\hline & AGGGCGACACCCTGGTGAA- & $d 15$ & 5 & \\
\hline & AGGGCGACACCCTGGTGAACCGCATCGA-------CT & $d 15$ & 1 & \\
\hline \#31-2 & AG-1 & d54 & 24 & $24 / 24$ \\
\hline \multirow[t]{3}{*}{ \#11 } & AGGGCGACACCCTGGTGAACCGCATCGAGCTGAAGGGCATCGACT & - & 11 & $19 / 30$ \\
\hline & AGGGCGACACCCTGGTGAACCGCATCGA--------CT & d15 & 17 & \\
\hline & AGGGCGACACCCTGGTGAACCGCATCGAGCTTGAAGGGCATCGAC & i1 & 2 & \\
\hline \multirow[t]{2}{*}{ \#18 } & 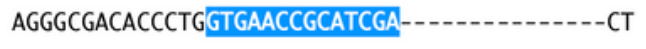 & d15 & 4 & $8 / 8$ \\
\hline & AGGGC-1 & $d 54$ & 4 & \\
\hline \multirow[t]{4}{*}{$\# 47-2$} & AGGGCGACACCCTGGTGAACCGCATCGAGCTGAAGGGCATCGACT & - & 4 & $26 / 30$ \\
\hline & 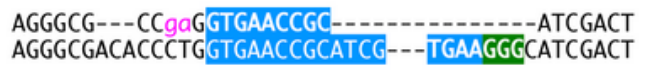 & $\begin{array}{l}\mathrm{d} 18 \mathrm{r} 2 \\
\mathrm{~d} 3\end{array}$ & 1 & \\
\hline & AGGGCGACACCCTGGTGAACCGCATCG---IGAAGGGCATCGACT & d3 & 10 & \\
\hline & AGGGCGACACCCTGGTGAACCGCATCGAGCTTTAAGGGCATCGAC & i1 & 15 & \\
\hline \multirow[t]{6}{*}{$\# 80$} & AGGGCGACACCCTGGTGAACCGCATCGAGCTGAAGGGCATCGACT & - & 1 & $19 / 20$ \\
\hline & AGGGCGACACCCTGGTGAACCGCATCGAGCTG-----(48)---- & $d 48$ & 8 & \\
\hline & AGGGCGACACCCTGGTGAA- & d15 & 3 & \\
\hline & AGGGCGACACCCTGGTGAACCGCATCGA-----AGGGCATCGACT & d5 & 3 & \\
\hline & AGGGCGACACCCTGGTGAACCGCATCGAGCT--AGGGCATCGACT & $d 2$ & 3 & \\
\hline & AGGGCGACACCCTGGTGAACCGCATCGAGCTTGAAGGGCATCGAC & i1 & 2 & \\
\hline
\end{tabular}

*Efficiency of mutation = total number of mutated embryos/total number of embryos

\section{Figure 4}

Serial observation of somatic embryogenesis and regeneration from embryogenic tissue (ET) line \#42, exhibiting patchy GFP fluorescence under visible light $(\mathrm{a}-\mathrm{e})$ and blue light $(\mathrm{f}-\mathrm{j})$, in which pZK_FFCas9 vector containing the gRNA of GFP target \#1 driven by CjU6_\#2 promoter was introduced. (a, f) Day 0, ET 
line \#42 in the somatic embryo maturation medium. Bars, $5 \mathrm{~mm}$. (b, g) Day 39, induction of cotyledonary somatic embryos in the same medium. GFP fluorescence is partially quenched in the cell mass. Bars, 5 $\mathrm{mm}$. (c, h) Higher magnifications of the framed boxes in (b) and (g), respectively. White arrows indicate GFP-knock-out embryos. Red arrowheads indicate embryos with active GFP. Bars, $1 \mathrm{~mm}$. (d, e, i, j) Germination of cotyledonary somatic embryos at $7 \mathrm{~d}$ after culture on germination medium. (d, i) Embryo with active GFP. Bars, 2 mm. (e, j) GFP-knock-out embryo. Bars, 2 mm. (k) Genotype of regenerated somatic cotyledonary embryos from selected ET lines. The target region and PAM sequence are highlighted in blue and green, respectively. A deletion is denoted by "-." Letters in red and lower-case pink indicate inserted and replaced bases, respectively. $\mathrm{d \# ,} \mathrm{i \# ,} \mathrm{and} \mathrm{r} \#$ denote the number of bp deleted, inserted, and replaced at the target site, respectively.

a

\section{CiChlI}

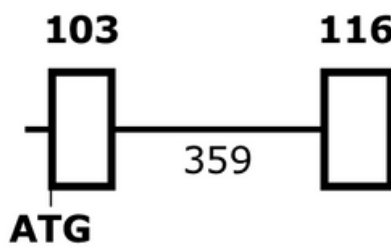

Target sequences

t1 t2 b

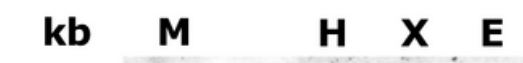

23.1-

9.41-

6.56-

4.36-

TGA

$2.02-$

\subsection{6-}
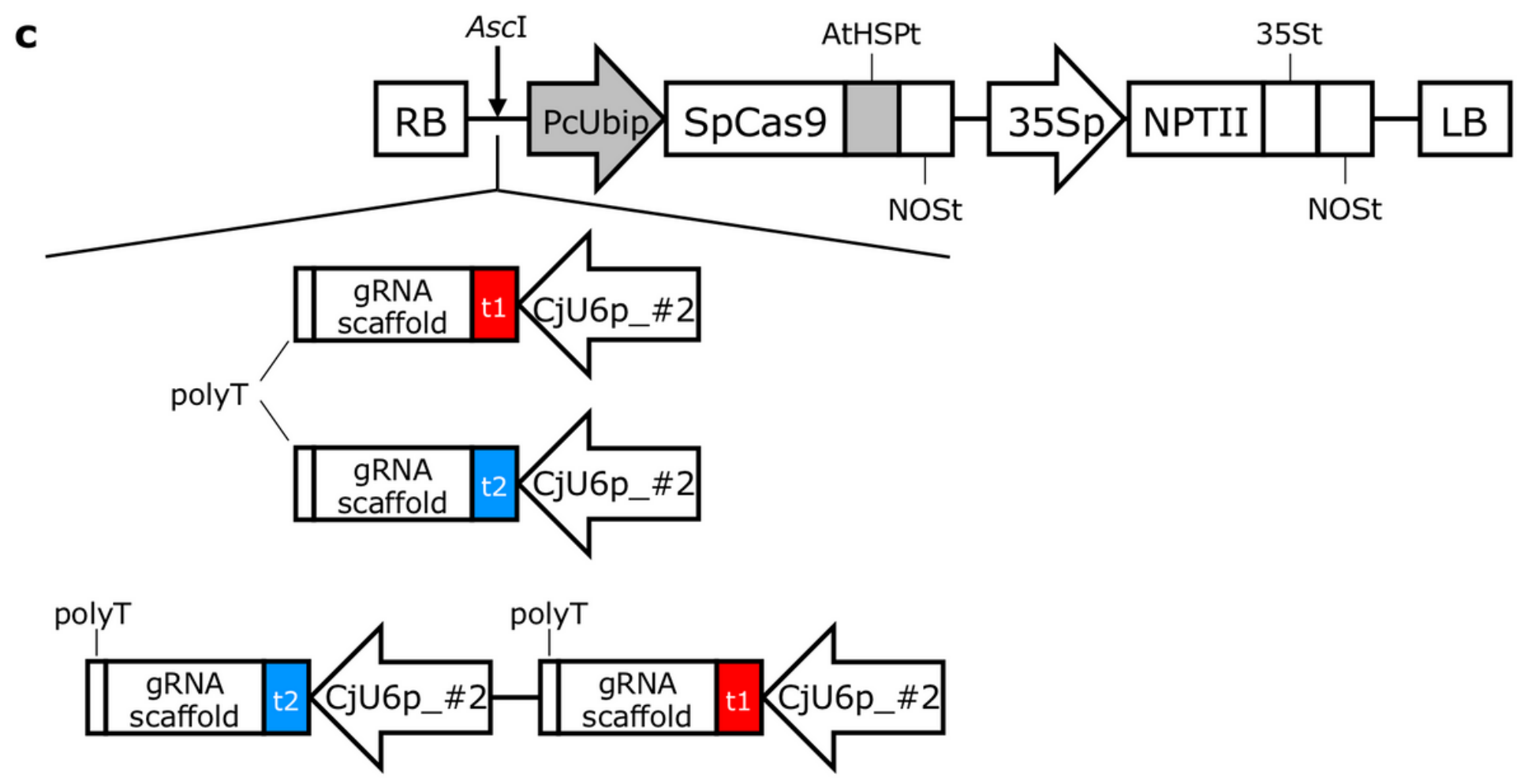


\section{Figure 5}

The structure of magnesium chelatase from Cryptomeria japonica. (a) Genome structure. Red letters show target 1 and blue letters show target 2, respectively. (b) Southern blot analysis of endogenous $C$. japonica magnesium chelatase subunit I (CjChlI). M; DIG-labeled $\lambda$-Hindlll marker, H; HindlII, X; Xhol, E; EcoRI. (c) Schematic representation of pCRG-SpCas9-derived CjChll-targeting vectors.
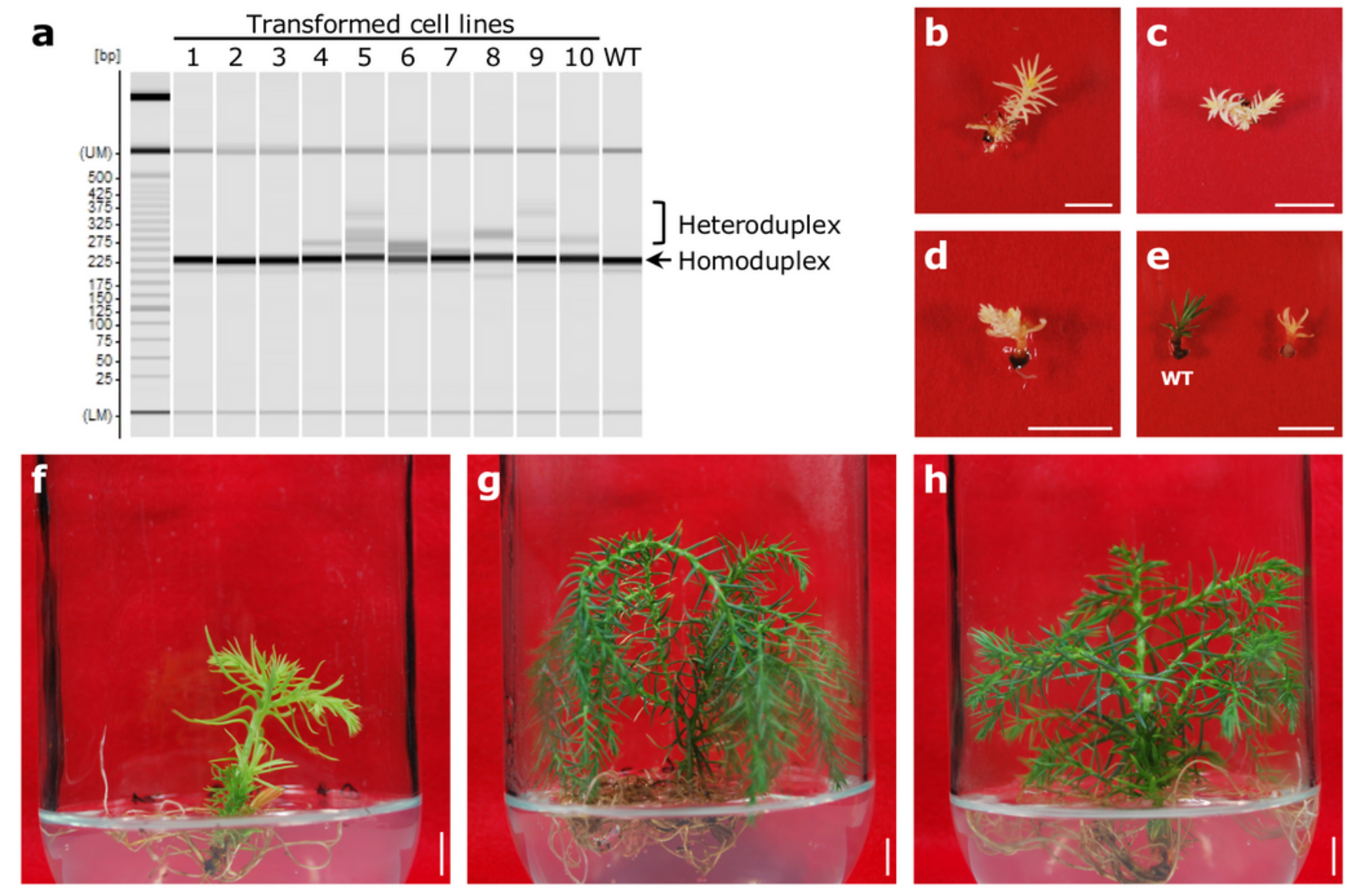

i

\begin{tabular}{|c|c|c|c|c|c|c|}
\hline Line & gRNA & Phenotype & Sequence of target site $\left(5^{\prime}-3^{\prime}\right)$ & Mutation & $\begin{array}{l}\text { No. of } \\
\text { clones }\end{array}$ & $\begin{array}{l}\text { Total } \\
\text { clones }\end{array}$ \\
\hline WT & - & Green & GTGATTGACCCAAAAATTGGAGGGGTTATGATAATGGGTGACCGTGG & - & - & - \\
\hline \#32-3_1 & t1 & Albino & $\begin{array}{l}\text { GTGATTGACC-1...-. GTGG } \\
\text { GTGATTGACCCAAAATTG-AGGGGTATGATAATGGGTGACCGTGG }\end{array}$ & $\begin{array}{l}\text { d33 } \\
\text { d1 }\end{array}$ & $\begin{array}{l}21 \\
6\end{array}$ & 27 \\
\hline \#11_1 & t2 & Albino & $\begin{array}{l}\text { GTGATTGACCCAAAAATTGGAG- } \\
\text { GTGATTGACCCAAAAATTGGAGGGGTATGATAATG-- }\end{array}$ & $\begin{array}{l}d 22 \\
d 8\end{array}$ & $\begin{array}{l}19 \\
13\end{array}$ & 32 \\
\hline$\# 11 \_2$ & t2 & Pale green & $\begin{array}{l}\text { GTGATTGACCCAAAAATTGGAGGGGTTATGATAATGGGTGACCGTGG } \\
\text { GTGATTGACCCAAAAATTGGAGGGGTTATGATAATGGGTIAtgaaGG }\end{array}$ & $\begin{array}{l}- \\
\text { r5 }\end{array}$ & $\begin{array}{l}7 \\
8\end{array}$ & 15 \\
\hline \#6-2_1 & $\mathrm{t} 1+\mathrm{t} 2$ & Albino & GTGATTGACCCAAAAATTGGGAGGGGTTATGATAATGGGTGACCGTG & i1 & 16 & 16 \\
\hline \#6-2_2 & $\mathrm{t} 1+\mathrm{t} 2$ & Albino & $\begin{array}{l}\text { GTGATTGACCCAAAAATTG-AGGGGTTATGATAATGGGTGACCGTGG } \\
\text { GTGATTGACCCAAAAATTG-AGGGGTIATGATAATGGGTGACCGTG }\end{array}$ & $\begin{array}{l}\text { d1 } \\
\text { d1i1 }\end{array}$ & $3^{13}$ & 16 \\
\hline \#6-2_3 & $\mathrm{t} 1+\mathrm{t} 2$ & Green & $\begin{array}{l}\text { GTGATTGACCCAAAAATTGGAGGGGTTATGATAATGGGTGACCGTGG } \\
\text { GTGATTGACCCAAAAATTGGAGGGGTTATGATAATGGGTGA-(15)- }\end{array}$ & $\bar{d} 15$ & $\begin{array}{l}15 \\
1\end{array}$ & 16 \\
\hline
\end{tabular}


Phenotype associated with CRISPR/Cas9-mediated editing of endogenous Cryptomeria japonica magnesium chelatase subunit I (CjChll). (a) Heteroduplex mobility assay of 10 transformed embryogenic tissue (ET) lines with pCRG-SpCas9-derived CjChll-targeting vectors and a wild-type ET line \#13-8-12 (WT). The formation of DNA heteroduplexes was observed in putative genome-edited lines (\#4-10). Regenerated plantlets from putative genome-edited line pCRG-SpCas9-t1_\#32-3_1 (b), pCRG-SpCas9t2_\#11_1 (c), pCRG-SpCas9-t1 + t2_\#6-2_1 (d), pCRG-SpCas9-t1 + t2_\#6-2_2 (e), pCRG-SpCas9-t2_\#11_2 (f), and pCRG-SpCas9-t1 + t2_\#6-2_3 (g). A regenerated plantlet of wild-type ET line \#13-8-12 (h). The seedling on the left in (e) is WT line \#13-8-12. Bars, $1 \mathrm{~cm}$. Plantlets of $(b-d)$, and $(f-h)$ were grown for 231 days on a germination medium, whereas plantlets of (e) were for 45 days on a germination medium. (i) Mutation pattern in each genome editing line. Target site sequences $\mathrm{t} 1$ and $\mathrm{t} 2$ are highlighted in red and blue, respectively. Letters in red and lower-case pink indicate inserted and replaced bases,

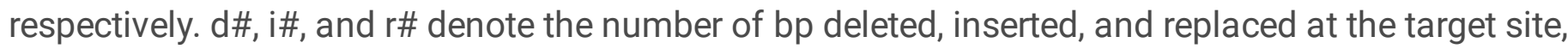
respectively.

\section{Supplementary Files}

This is a list of supplementary files associated with this preprint. Click to download.

- FigureS1.tiff

- Figures2.tiff

- Figures3.tiff

- Figures4.tiff

- FigureS5a.tiff

- FigureS5b.tiff

- FigureS5c.tiff

- Figures6.tiff

- Supplementarylmformationfinal.docx 